\title{
Magnetic multilayers on nanospheres
}

\author{
MANFRED ALBRECHT ${ }^{1 *}$, GUOHAN HU ${ }^{2}$, ILDICO L. GUHR ${ }^{1}$, TILL C. ULBRICH ${ }^{1}$, \\ J OHANNES BONEBERG ${ }^{1}$, PAUL LEIDERER ${ }^{1}$ AND GÜNTER SCHATZ ${ }^{1}$ \\ IUniversity of Konstanz, Department of Physics, D-78457 Konstanz, Germany \\ ${ }^{2}$ Hitachi San J ose Research Center, 650 Harry Road, San J ose, California 95120, USA \\ *e-mail: manfred.albrecht@uni-konstanz.de
}

T hin-film technology is widely implemented in numerous applications ${ }^{1}$. Although flat substrates are commonly used, we report on the advantages of using curved surfaces as a substrate. The curvature induces a lateral film-thickness variation that allows alteration of the properties of the deposited material ${ }^{2,3}$. Based on this concept, a variety of implementations in materials science can be expected. As an example, a topographic pattern formed of spherical nanoparticles ${ }^{4,5}$ is combined with magnetic multilayer film deposition. Here we show that this combination leads to a new class of magnetic material with a unique combination of remarkable properties: The so-formed nanostructures are monodisperse, magnetically isolated, single-domain, and reveal a uniform magnetic anisotropy with an unexpected switching behaviour induced by their spherical shape. Furthermore, changing the deposition angle with respect to the particle ensemble allows tailoring of the orientation of the magnetic anisotropy, which results in tilted nanostructure material.

In modern magnetic recording materials, the'superparamagnetic effect' has become increasingly important as new magnetic harddisk-drive products are designed for higher storage densities. This phenomenon is based on the fact that the thermal stability of the magnetization orientation of magnetically decoupled grains of the recording material scales with magnetic anisotropy strength and grain volume ${ }^{6,7}$. Thus, the magnetization of the grains may become unstable due to thermally activated fluctuationsas the grains become smaller.O neapproach to delay superparamagnetism isto compensate the decrease in grain size by increasing the magnetic anisotropy. In this regard, nanoparticle media ${ }^{6,8}$, where two-dimensional arrays of monodisperse nanoparticles with high magnetic anisotropy are used, is assumed to be the ideal future magnetic recording material. However, key requirements like control of the magnetic anisotropy orientation along with magnetic domain isolation have not been achieved so far. Here we present an approach that leads to the required properties by combining a nanoscale topographic pattern

Figure 1 Magnetic images of self-assembled particle arrays after $\mathrm{Co} / \mathrm{Pd}$ film deposition. a, Schematic picture of the film deposited onto nanospheres. MFM images are presented for arrays with a particle size of $\mathbf{b}, 310 \mathrm{~nm}, \mathbf{c}, 110 \mathrm{~nm}$, and $\mathbf{d}$, $50 \mathrm{~nm}$. The images were taken after demagnetizing the samples.
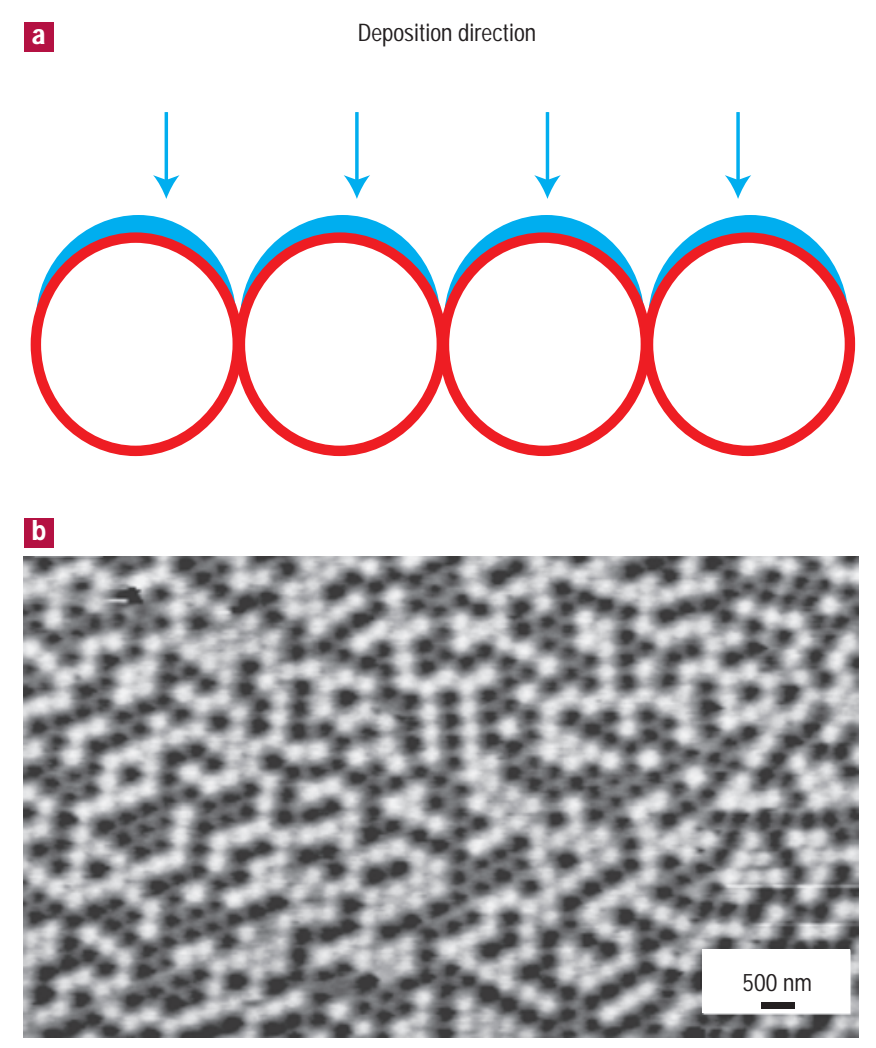

c

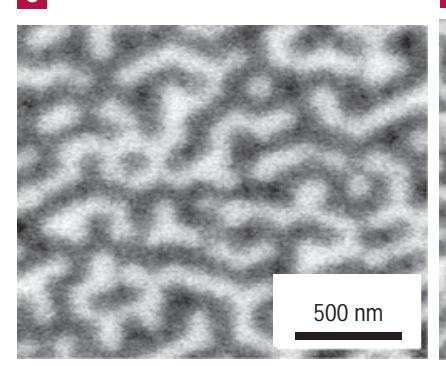

d

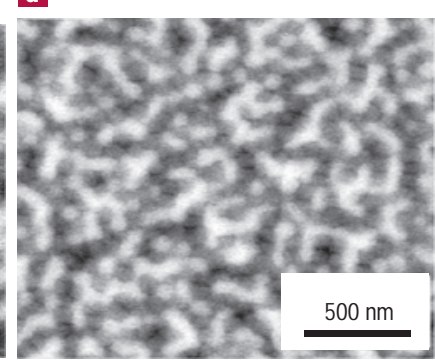




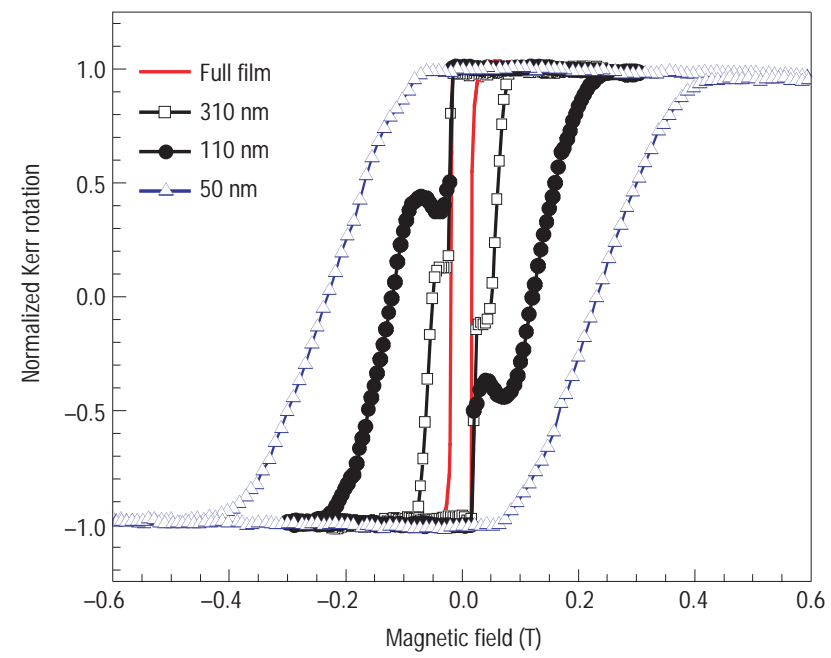

Figure 2 Polar MOKE hysteresis loops. Data obtained after Co/Pd film deposition on different particle arrays with particle diameters from $50 \mathrm{~nm}$ to $310 \mathrm{~nm}$, and for comparison on a plain glass substrate.

with magnetic multilayer film deposition.

For this purpose, densely packed two-dimensional arrays of monodisperse spherical polystyrene particles in the size range from 50 to $310 \mathrm{~nm}$ are formed by self-assembling upon slow evaporation of a solvent under ambient conditions. Such particle monolayers are typically used as a two-dimensional deposition mask for nanostructure fabrication followed by mask removal 4,5 . However, in our studies the monolayer itself acts as a topographic pattern as illustrated in Fig. 1a. The magnetic film deposition was carried out in a molecular beam epitaxy chamber operating at a base pressure of $2 \times 10^{-10} \mathrm{mbar}$. Co/Pd multilayer films were deposited at room temperature on the prepared particle monolayer by evaporation of $\mathrm{Co}$ and $\mathrm{Pd}$ from two electron-beam sources. In our studies, a multilayer consisting of a $[\mathrm{Co}(0.3 \mathrm{~nm}) / \mathrm{Pd}(0.8 \mathrm{~nm})]_{8}$ stack deposited on a 3-nm-thick Pd seed layer was used for the samples and covered by an additional $0.8-\mathrm{nm}$-thick Pd layer to prevent oxidation. For this stacking it is known that strong magnetic anisotropy pointing perpendicular to the surface can be obtained ${ }^{9,10}$. By film deposition on the particle monolayer, two different arrays of magnetic nanostructures are formed. First, the densely packed particle monolayer acts as a deposition mask leading to isolated magnetic nanostructures deposited directly on the glass substrate. Second, the tops of each spherical particleare covered by the deposited magnetic multilayer film forming spherically shaped nanostructures referred to as magnetic caps in the following paragraphs.

The samples were imaged by magnetic force microscopy (M FM ).[Author: Please give make and model of equipment used.] O wing to the large distance towards the substrate, only the magnetic caps give rise to a contribution in the M FM signal. Figure 1 shows magnetic images of arrays with particle sizes of (b) $310 \mathrm{~nm}$, (c) $110 \mathrm{~nm}$, and (d) $50 \mathrm{~nm}$. A uniform white or dark contrast is seen on each particle, which suggests that the nanostructures are in an isolated magnetic singledomain state with the magnetization pointing perpendicular to the substrate surface. Serpentinelike magnetic domain patterns were observed on all images following clearly the topographic pattern corresponding to the minimum of the magnetostatic energy for a hexagonal lattice of exchangeisolated particles with perpendicular magnetic anisotropy. The mechanism
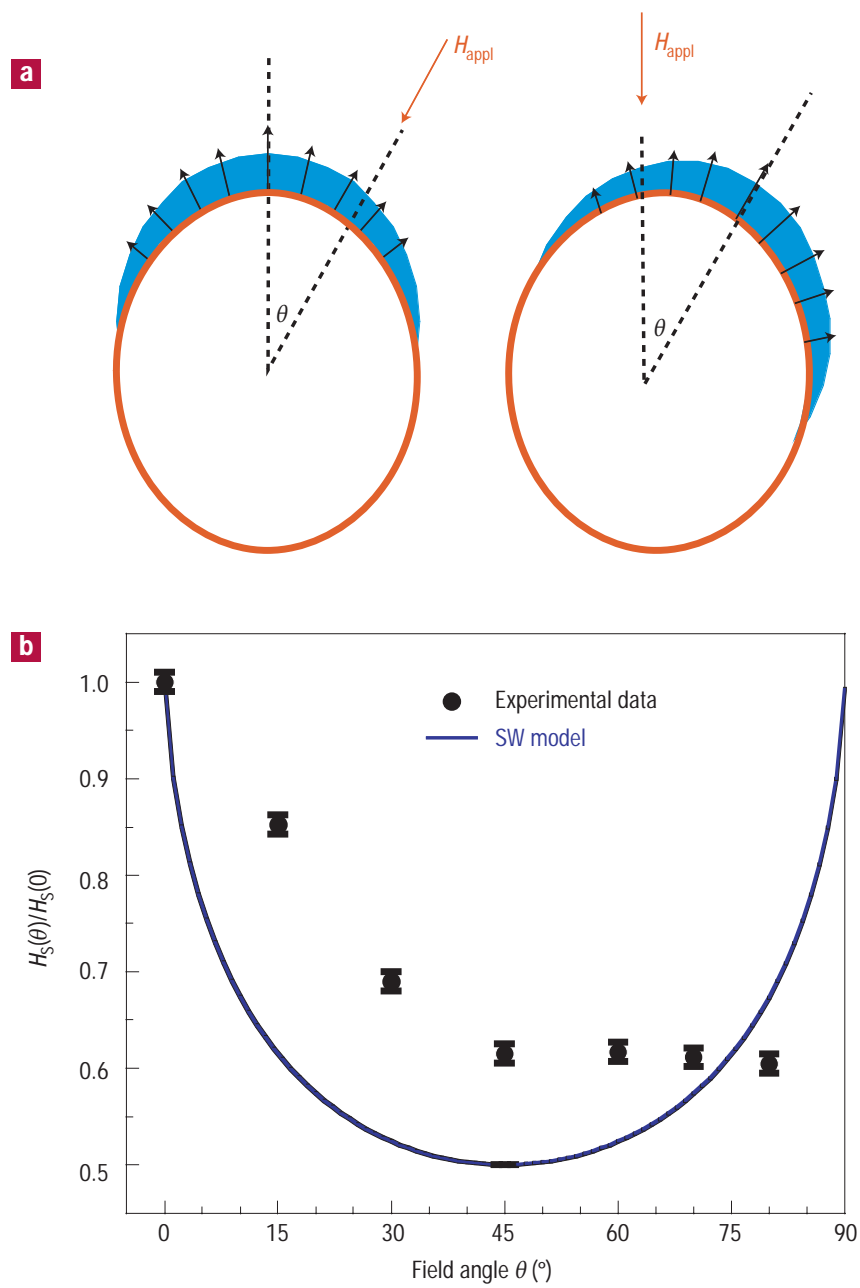

Figure 3 Switching field $\left(\mathbf{H}_{3}\right)$ as a function of the angle $(\theta)$ of the applied field $\left(\mathbf{H}_{\mathrm{ppp}}\right)$. [Author: OK?] a, Schematic of a magnetic film deposited on a nanosphere showing the anisotropy distribution indicated by arrows. $\mathbf{b}$, Switching field as a function of applied field angle for an array with $50-\mathrm{nm}$ particles (black dots). The angular dependence based on the Stoner-Wohlfarth (SW) model is shown as a blue line for comparison. $\mathbf{c}$, The average anisotropy axis can be tuned to the required angle by changing the deposition direction.

of magnetic isolation of the caps is related to two effects. First, each spherical particle has only six small contact areas between neighbouring spheres that can transfer limited exchange interaction between the magnetic caps. Second, the curvature of the surface plays an important role for the deposited film thickness. Assuming an evaporation direction perpendicular to the substrate plane, the spherical surface of the particle will lead to a reduction in $\mathrm{Co}$ and $\mathrm{Pd}$ thicknessalong thesurface, which in turn suppressesferromagnetism in the Pd/Co system towards the contact area between neighbouring particles. This mechanism of domain isolation is very effective for an extremely thin Co thickness for instance of only one atomic monolayer and should hold for even smaller particle sizes in the 10-nm range.

The M FM studies were supplemented by magneto-optical Kerr effect (M OKE) measurements using a focused laser beam with a spot size of about $40 \mu \mathrm{m}$ and superconductive quantum interference device (SQUID) magnetometry.[Author: Please give make and 
model of equipment used.] Figure 2 shows polar M OKE hysteresis loops obtained after $\mathrm{Co} / \mathrm{Pd}$ film deposition on different particle arrays and, for comparison, on a plain glass substrate. In this polar geometry an external magnetic field perpendicular to the substrate surface is applied while measuring the magnetization component (given by the Kerr rotation) along this direction. Note that the recorded MOKE signal of the particle arrays is only stemming from the magnetic island caps as confirmed by MFM and SQUID. The full film reveals perpendicular magnetic anisotropy $\left(\mathrm{K}_{\mathrm{u}}=0.4 \mathrm{MJ} \mathrm{m}^{-3}\right)$ [Author: Please define $\mathrm{K}_{\mathrm{u}}$ ] with a small coercivity of $30 \mathrm{mT}$, and a narrow reverse field distribution. In contrast, for the spherical nanostructures, the coercivity as well as the width of the reverse field distribution increases with decreasing particle size. This behaviour is similar to results reported on arrays fabricated by electron-beam lithography, and is a result of a more coherent rotational switching process ${ }^{11,12}$. H owever, a perpendicular magnetic anisotropy isstill present and dropsonly by afactor of 1.5. In addition, some of the M-H [Author: Please define M and H (magnetizationfield?)] loops show an additional sharp reversal at about $30 \mathrm{mT}$, which is due to areas without particles revealing full film properties. The anomalous Kerr signal obtained in the field range between 30 and $40 \mathrm{mT}$ for the 110-nm particle array is due to an interference phenomenon ${ }^{13}$ between the magneto-optical responds and the topographic pattern, and not related to themagnetization, as verified by SQUID.

Another remarkable feature is the magnetic anisotropy present in the spherically shaped caps. Perpendicular magnetic anisotropy observed in Co/Pd multilayer films is mainly given by the interface anisotropy between $\mathrm{Co}$ and Pd layers, which points perpendicular to the interface layer ${ }^{9,10}$. Therefore, film deposition on a spherical surface $^{2}$ will in general result in a spread of the anisotropy axis across the surface with the average anisotropy axis pointing along the deposition direction. However, not only will the angle of the anisotropy axis gradually change across the surface, but also its strength will vary according to the layer thickness, 10 as depicted in Fig. 3a. These variations will have a drastic impact on the switching mechanism. Studies on the angular dependent switching behaviour were performed by remanent hysteresis loops for an array of 50-nm particles. In these measurements the sample is first saturated and then a magnetic reverse field is applied. The so called switching field $\mathrm{H}_{\mathrm{s}}$ is the field that is required to reverse the magnetization direction of half of thenanostructures in the array, and is plotted as a function of applied field angle $\theta$ between the magnetic field and the average anisotropy axis. Whereas magnetic single-domain nanostructures with uniaxial magnetic anisotropy follow a Stoner-Wohlfarth switching behaviour ${ }^{14}$, the magnetic caps reverse in a rather unusual way as shown in Fig. 3b. Here, a rapid decrease of the switching field is found with increasing field angle up to an angle of about $45^{\circ}$ and between $45^{\circ}$ and $80^{\circ}$ it remains almost unchanged. Thus, the sensitivity of the switching field to $\theta$ and the switching field itself is at its minimum over a large range of angle. This remarkable behaviour is mainly given by the presence of different angle configurations between anisotropy axis and applied field across the caps, as confirmed by micromagnetic simulations ${ }^{15}$, which will be presented in detail in a forthcoming publication.

In order to use the advantages of the angle-dependent switching process, uniform fields with a tilt of more than $45^{\circ}$ are required. However, the design of a head capable of generating a uniform and tilted field is challenging. In contrast, use of tilted media ${ }^{16,17}$ is a possible way to solve the above problem. By adjusting the average anisotropy direction in the tilted media, $\theta$ can betuned to the points where the sensitivity of the switching field is near to its minimum. M oreover, as the switching field is al so near to its absolute minimum at these points, thermally stable high $\mathrm{K}_{\mathrm{u}}$ materials are writable. Here in the presented medium, $\theta$ can be tuned to the required

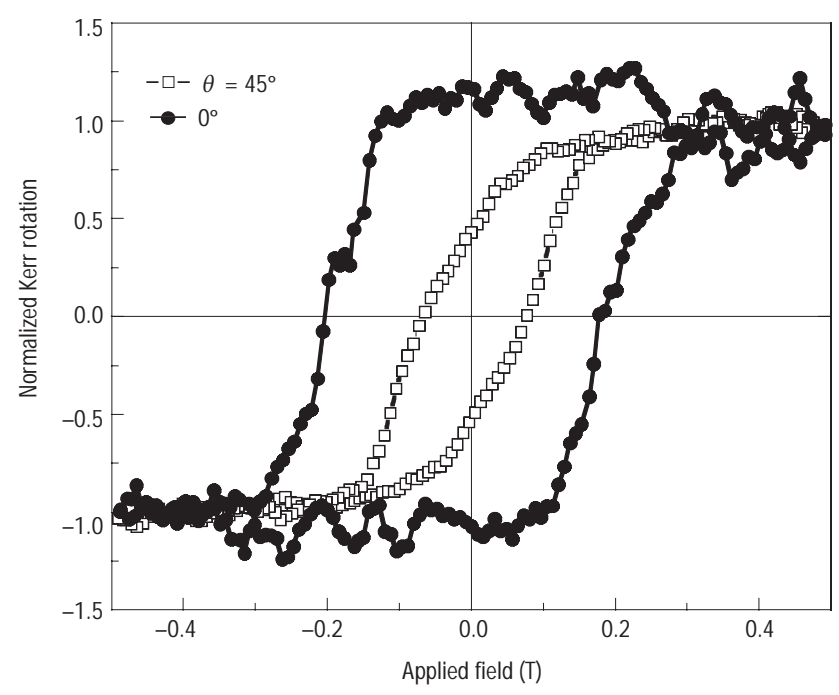

Figure 4 MOKE hysteresis loops. Data taken under different field angles for an array of particles with a diameter of $270 \mathrm{~nm}$ where the deposition angle was tilted by $45^{\circ}$ with respect to the substrate normal resulting in a tilt of the average anisotropy axis by $45^{\circ}$. Note that $\theta=0^{\circ}$ indicates that the applied magnetic field and the average anisotropy axis are pointing in the same direction, however, both are tilted by $45^{\circ}$ with respect to the substrate normal.

angle by changing the deposition direction as illustrated in Fig. 3c. To demonstrate its feasibility, a deposition angle of about $45^{\circ}$ with respect to the surface normal of the substrate was used on an array with a particle size of $270 \mathrm{~nm}$. Thus, an average anisotropy axis tilted by $45^{\circ}$ with respect to the surface normal is expected. $\mathrm{M}-\mathrm{H}$ loops were measured at different applied field angles to determine the magnetization and therefore the easy axis direction. A square hysteresis loop was found under an angle $\theta=0^{\circ}$, which clearly confirms an easy axis direction, whereas at $\theta=45^{\circ}$ an off-axis loop was obtained as shown in Fig. 4.

The presented concept leads to a realization of tilted nanostructure media, which offer new advances in achieving higher densities following 'M oore's Law' in future storage devices. M oreover, a variety of further implementations of curved surfaces in materials science can be expected. Any material with strongly thickness-dependent properties, for instance superconductors or optical materials, is a potential candidate.

Received 1 September 2004; accepted 3 J anuary 2004; published XX xxxx 2005

\section{References}

1. Elshabini-Riad, A. \& Barlow, F. D. Thin-Film Technology Handbook (M cGraw-Hill, New York, 1997).

2. Chen, Y. J. et al. Periodic magnetic nanostructures on self-assembled surfaces by ion beam bombardment. J. Appl. Phys. 91, 7323-7325 (2002).

3. Chalastaras, A. et al. GM R multilayers on a new embossed surface. IEEE Trans. M agn. 40, 2257-2259 (2004).

4. Fischer, U. C. \& Zingsheim, H. P. Submicroscopic pattern replication with visible light. J. Vac. Sci. Technol. 19, 881-885 (1981).

5. Deckman, H. W. \& Dunsmuir, J. H. Natural lithography. Appl. Phys. Lett. 41, 377-379 (1982)

6. Moser, A. et al. Magnetic recording: advancing into the future. J. Phys. D 35, R157-167 (2002)

7. Weller, D. \& M oser, A. Thermal effect limits in ultrahigh-density magnetic recording. IEEE Trans. Magn. 35, 4423-4439 (1999).

8. Sun, S., Murray, C. B., Weller, D., Folks, L. \& Moser, A. M onodisperse FePt nanoparticles and ferromagnetic FePt nanocrystal superlattices. Science 87, 1989-1992 (2000).

9. Carcia, P. F., M einhaldt, A. D. \& Suna A. Perpendicular magnetic anisotropy in Pd/Co thin film layered structures. Appl. Phys. Lett. 47, 178-180 (1985).

10. Suzuki, T. Coercivity mechanism in ( $\mathrm{Co} / \mathrm{Pt})$ and $(\mathrm{Co} / \mathrm{Pd})$ multilayers. Scripta M etall. M ater. 33, 1609-1623 (1995)

11. Hu, G. et al. M agnetic and recording properties of $\mathrm{Co} / \mathrm{Pd}$ islands on prepatterned substrates. J. Appl. 


\section{LETTERS}

Phys. 95, 7013-7015 (2004).

12. Landis, S., Rodmacq, B. \& Dieny, B. M agnetic properties of Co/Pt multilayers deposited on silicon dot arrays. Phys. Rev. B 62, 12271-12281 (2000).

13. Bardou, N . et al. Light diffraction effects in the magneto-optical properties of $2 \mathrm{D}$ arrays of magnetic dots of $\mathrm{Au} / \mathrm{Co} / \mathrm{Au}(111)$ films with perpendicular magnetic anisotropy. J. M agn. M agn. M ater. 148, 293-294 (1995).

14. Stoner, E. C. \& Wohlfarth, E. P. A mechanism of magnetic hysteresis in heterogeneous alloys. Philos. Trans. R. Soc. Lond. A 240, 599-642 (1948).

15. Scholz, W. et al. Implementation of a high performance parallel finite element micromagnetics package. J. M agn. M agn. M ater. 272, 693-694 (2004).

16. Zou, Y. Y., Wang, J. P., Hee, C. H. \& Chong, T. C. Tilted media in a perpendicular recording system for high areal density recording. Appl. Phys. Lett. 82, 2473-2475 (2003).

17. Kai-Zhong, G. \& Bertram, H. N. Transition jitter estimates in tilted and conventional perpendicular recording media at $1 \mathrm{~Tb} / \mathrm{in}^{2}$. IEEE Trans. M agn. 39, 704-709 (2003).

Acknowledgements

This work was supported by the Deutsche Forschungsgemeinschaft through SFB 513 and the

Emmy Noether program.

Correspondence and requests for materials should be addressed to $M$ A

Competing financial interests

The authors have declared that they have no competing financial interests. 